\title{
Design and Implementation of AR Electronic Map System Based on IOS
}

\author{
Mingyuan Zhang ${ }^{1}$, Zhongliang $\mathrm{Cai}^{2}$, Meng $\mathrm{He}^{2}$ \\ ${ }^{1}$ School of Geodesy and Geomatics, Wuhan University, China. \\ 2 School of Resource and Environmental and Sciences, Wuhan University, China. \\ * Corresponding author. Email: zlcai@whu.edu.cn \\ Manuscript submitted June 30, 2017; accepted August 12, 2017. \\ doi: 10.17706/jsw.12.10.807-815
}

\begin{abstract}
The rapid development of LBS mobile application has become an essential tool for people to travel and brought great convenience to people's life. Under such circumstances, the application of real technology in mobile ends has emerged as a new trend. However, the existing LBS applications do not do enough for the identification of spatial targets, not intuitive and guidance, and need a new way to identify space targets.Using augmented reality technology can greatly enhance the user experience index of LBS applications, and give people the feeling of being immersive.This paper developed a mobile electronic map information system based on IOS applications with Augmented Reality (AR) technology, catering to the requirement. Traditional functions of mobile electronic map, such as querying position, data updates within the system have been achieved. In addition, AR technology was applied to interact with the camera screen (real scene) for achieving a space target identification function.
\end{abstract}

Key words: Location service, augmented reality, real-time identify, dynamic display.

\section{Introduction}

Spatial information has become the basic element of modern digital city. People in their daily lives are exposed or available information, of which $80 \%$ and space information is closely related. It can be said that more and more inseparable from the space information. With the rapid development of positioning technology, people can easily get their current location, but people are not satisfied with the simple coordinate information and want to get more around the geography-related information. So the location-based services (Referred to as LBS ) came into being.

With the continuous innovation of interactive technology, new ways of interaction continue to emerge, such as virtual reality, enhance the reality, intelligent wear equipment, people on the software interactive way put forward higher requirements. This change also occurs in location services, and the combination of Augmented Reality (AR) technology and location-based services is an alternative to improving the interaction experience.

As the cognitive needs of the continuous development and desire to a more realistic way to know their surrounding information world, the spatial target display and query the demand is more urgent. This paper developed a mobile electronic map information system based on IOS applications with Augmented Reality (AR) technology, catering to the requirement.

The rest of this article is organized as follows. We discuss the overall application analysis in Part 2. We proceed to Part 3 to proceed with the overall system architecture and system implementation in detail. In 
Section 4, we summarize the research work done in this paper and look forward to future research.

\section{Application Analysis}

This paper focuses on the identification of AR technology and space targets based on location services.

\subsection{Development Model}

The software uses the Objective-c language implementation, calls the Amap API to build the application framework, uses ARKit as the AR engine, and provides the space object identification and display service on the basis of the location service.

IOS is one of the most popular mobile operating systems [1]. The development of IDE Xcode provides a very high support language for standard $\mathrm{C}++$ through its proposed programming for Objective-C [2]. It can call the standard $\mathrm{C}++$ wrapper library functions, whether it is static or dynamic library. It also supports the mixed coding of Objective-C and standard $\mathrm{C}++[3]$.

Amap open platform offers 2D, 3D, satellite variety of map form for developers to choose. It can be provided by the high open platform API to complete the map to build the work.

The commonly used AR development tools are ARToolKit, Vuforia, AR and ARKit. Since ARKit is more suitable for the iOS platform than the other two toolkits, with the iOS built-in positioning service framework, real-time coordinates can be obtained, and the display position of the objects in the screen can be updated in real time. The development kit supports all directions and captures different realistic scene targets as the camera's orientation changes, and changes the display status of the virtual object in real-time according to different directions and distances. In the AR display mode, a friendly interaction is provided for users, such as users who can click on objects displayed in the picture for more detailed information. At the same time, the toolkit has built-in radar views that developers can easily call. Support multi view overlay display, and have good customization.

\subsection{Research Content}

\subsubsection{Experimental data acquisition}

The software uses the data is mainly the background data and POI data of these two categories of data. The main function of map is used to display the data as the basis for the use of GIS to carry out some application. The set of spatial objects constitutes POI data, and the user searches for the target elements in the server database by keyword. The main data used in this experiment is the Amap online resources, maps and POI search is an online resource for mobile intelligent networking equipment, using Wifi auxiliary positioning and the introduction of iOS system for the development of the location service framework of Core Location. At the same time, the device acceleration is required. The iPad device has three directions of acceleration. According to the acceleration component in the three directions, the moving direction of the device can be calculated to obtain the moving direction data. The space target in the experiment can sense device movement. When the device is moving, the space object requires change the display position dynamically in real time.

\subsubsection{Real-time identification of space objectives}

With the development of technology and the increasing demand of people, the requirement of real-time identification of space targets is demanding. Because the user equipment display screen size is limited, can not be loaded once all the space target, or the display space of all attributes, requires a combination of related applications for screening, extraction of the user may be interested in the space target and relevant attribute information. Therefore, buffer analysis is necessary.Firstly, space target information storage determines what information can be transmitted to the client from the server, relates to the characteristics 
of space target space, information and data points of interest, and inverse geographic classification encoding encoding, POI encoding geographic data acquisition and database storage process. Spatial data stored in the server needs to be transmitted to the client. This process involves spatial information transmission technology, and wireless LBS mainly uses wireless networks to communicate. The user can get to the current location coordinates, the coordinates of points in the current need to establish a buffer zone, then query in this buffer, the query to the target point after calculating the azimuth angle, the spatial distribution of spatial target, and then identifies the region, identify the target in the camera.At present, the development of the Augmented Reality (AR) technology based on the logo is more mature. Through the C / $S$ mode [4], the identity database is set up on the server and it is transmitted to the mobile device through the mobile communication network or Wifi. So the mobile device can easily realize the Augmented Reality (AR) application.

\subsubsection{Dynamic display of spatial objects}

As a mobile electronic map system using AR technology, this paper use the camera to capture the real scene as the AR basis scene [5]. The spatial object is registered in the real camera scene. The virtual object is registered in the real camera scene according to the geographical coordinates according to the certain spatial distribution. According to the registration effect, the LBS application necessary map added to the scene, so that the map and camera scene combination, camera changes and map view changes need to do synchronization. In AR applications, two views are usually used to display spatial objects, one to display the fusion effect between virtual targets and real scenes, and a radar like circular view to display spatial target profiles.

\subsubsection{The realization of traditional electronic map service}

As a mobile electronic map system, the system has also developed and implemented the traditional functions of mobile electronic maps such as location, query and path planning through Amap API. Compared with the traditional mobile electronic map, the system will combine the camera scene and electronic map dynamic display, and is equipped with a small radar at the bottom, completely changed the traditional way of reading a map, intuitive and efficient. For example, people can do so by pointing the camera in one direction so that they can know the name and property of the building after the current building is blocked.

\section{System Design and Implementation}

\subsection{The overall design of the system}

The mobile electronic map system is divided into four modules, which are data module, data transmission module, display control module, display application module. Among them, the specific application layer includes many application functions. The following is the overall architecture display:

\subsection{Specific Implementation}

\subsubsection{Experimental data acquisition}

This experiment is implemented in Objective-c language. It builds the application framework by calling the Amap API, and uses ARKit as the AR engine. It provides spatial target identification and display services on the basis of location-based services. From the Amap LBS open platform official website download Amap iOS, SDK, V2.4.2 version. This paper download the 3D version, which can provide three-dimensional services, full-featured, excellent results. After downloading the development package requires configure, there are two main configurations, automatic configuration and manual configuration. In this paper the 
choice is manual configuration. First, a Single View application is built in Xcode. Device selects iPad. The project name is MyPOIMarker. These are the following steps:

Introduce the map library. Add the extracted MAMapKit.framework file to the project.

Introduce the AMap.bundle resource file. AMap.bundle resource file stored in the positioning, default pin view and other images, you can use these resources to develop the picture.

Introduced into the system library. According to the list provided by the official website, the required system libraries are introduced.

Engineering environment configuration. Add -ObjC to TARGETS-> Build Settings-> Other Linker Flags.

Use the ARKit library to develop an AR application to clone an example demo from GitHub named "ios-arkit" with the name calonso and ARKit Example. After cloning down, copy the ARKit folder to the MyPOIMarker project directory as the source code for the project AR engine.

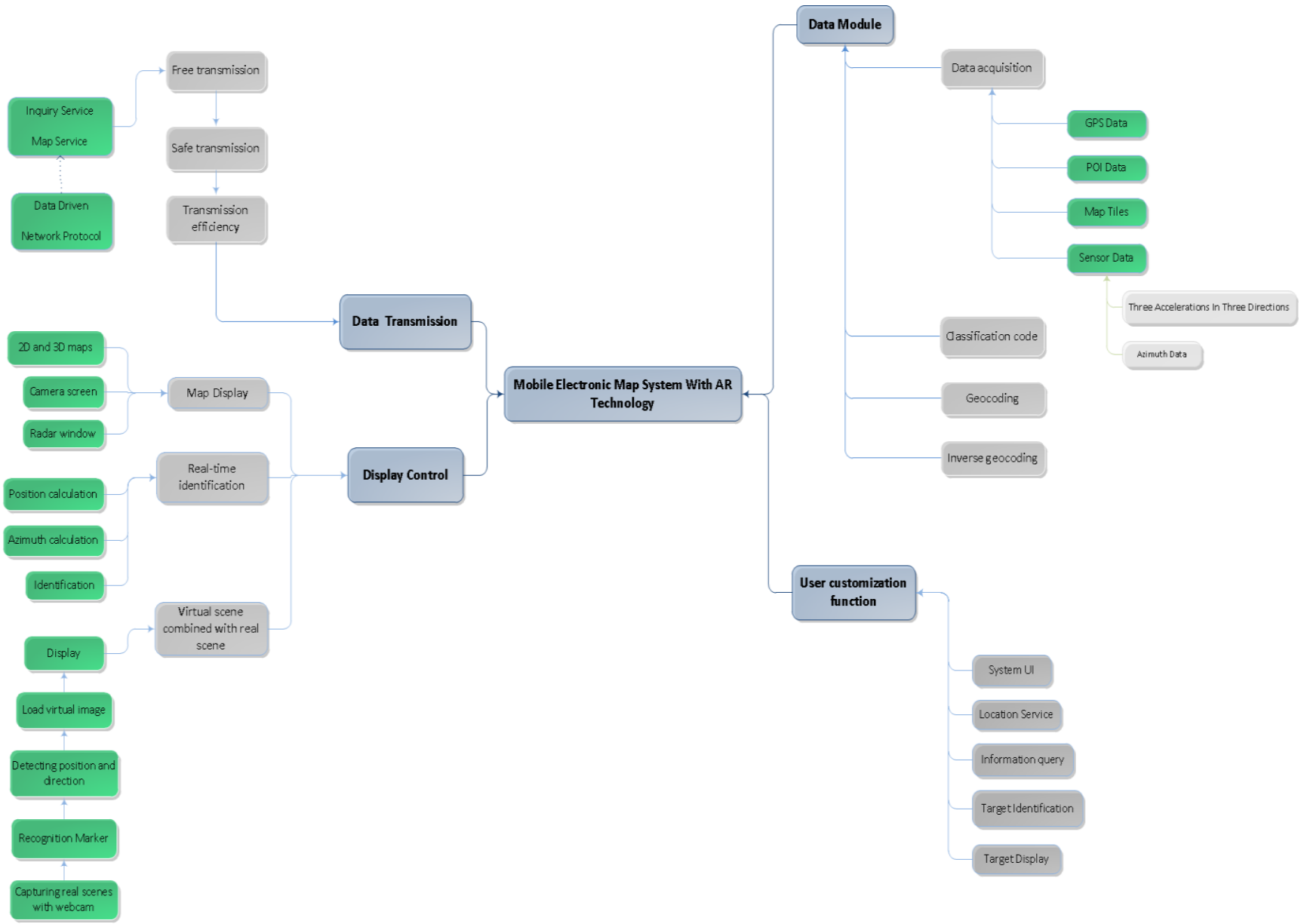

Fig. 1. Overall design.

\subsubsection{Basic class structure design of the system}

The overall use of iOS under the popular MVC model. The system consists of two MVC modes, namely the system overall mode and the ARKit mode. ViewController is the core of the entire application system. It plays a key role in the application and can handle views and models.

The view consists of two tables, the table view and the map view. The table view is responsible for enumerating the POI collection. The map view is used to display the map. UITableView is a class in Cocoa Touch, and MAMapView is a class in the Amap API.

There are two main models, namely NSArray and NSMutableArray. NSArray stores the POI search results set. The array element is an object of the Amap POI class that stores POI various information. 
NSMutableArray stores the map annotation, which is the map element used to display the search for the POI target and represents a pin on the map. The tag element is the MAPointAnnotation class object, with the name and address information of the POI, respectively, as the caption and subtitle of the tag.

ARKit is the AR Engine development kit. The core class is ARKitEngine, used to operate the entire engine. The model is the geographic coordinate class of the AR target object, which stores the data object, the radar radius, the tilt angle, and the azimuth angle. The view mainly includes the AR target object view and the auxiliary view of the radar view.

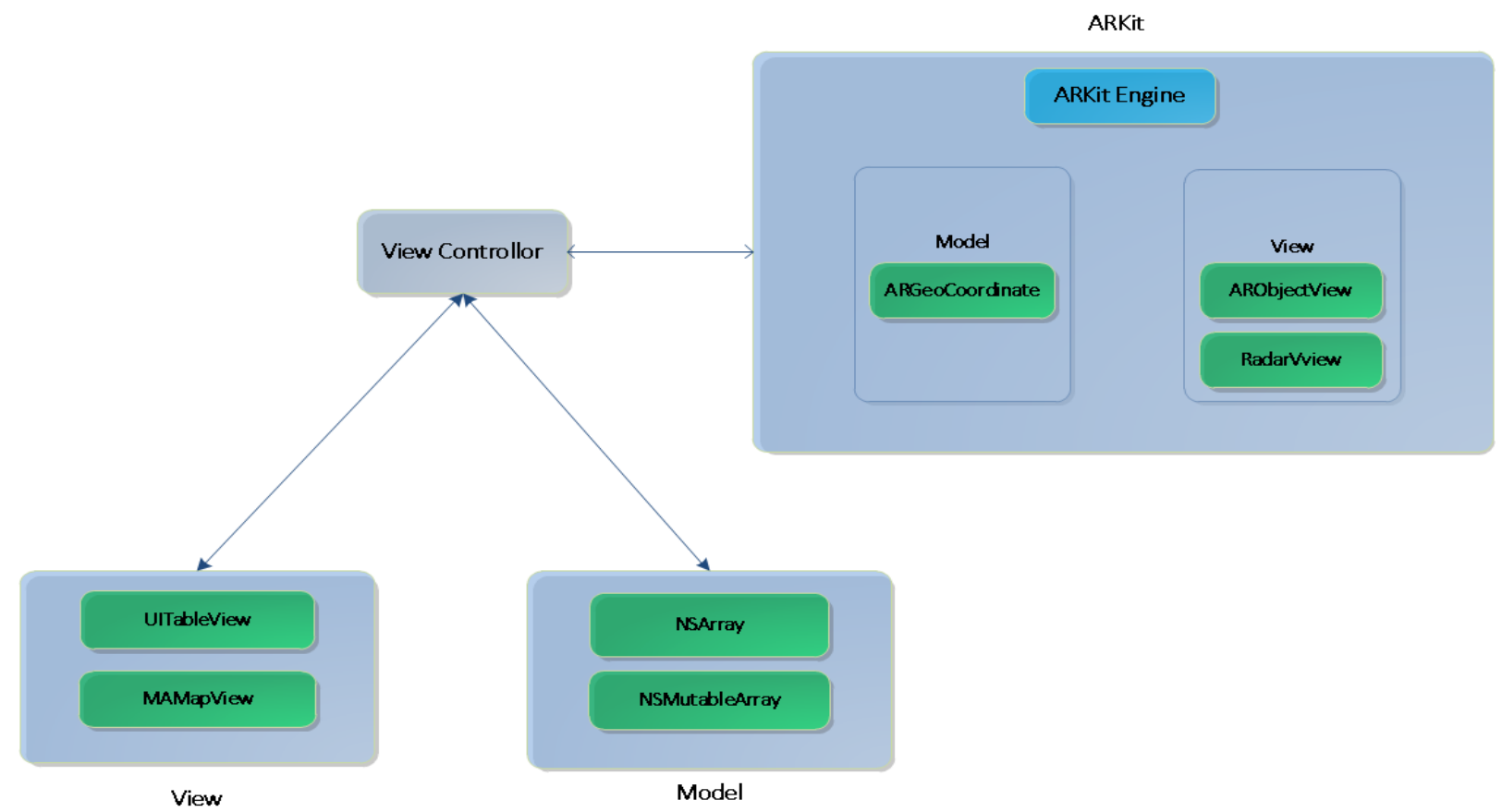

Fig. 2. System MVC class structure.

\subsubsection{Spatial object display mode}

POI records are searched for from Amap POI database server based on keywords. These POI records are not a single data, but a collection of data structures that contain a lot of rich information that needs to be displayed on a limited number of mobile devices. And the way to be able to do practical significance.

First of all, the whole simulation of the space target is carried out. This article studies a way to use icons and text. The overall shape of the object is rectangular and the length of the rectangle is slightly larger so that more text can be accommodated in one line. Divide the rectangle into two columns, which are used to display the most important two information of the POI, the name and the distance.

Nilsson et al[3] proposed simulation object display, using tagging method to display objects outside the display. Because of the complexity of the actual environment, and a variety of man-made landscape, more in a continuous structure. In this paper, the visualization of the spatial object in the display screen adds the distance factor and captures the space object to obtain the distance between the object and the current position. According to the distance adjusting object size, with a user near the small effect, closer to the real scene.

\subsubsection{Information transmission}

The system uses wireless LBS integrated application platform. The platform is a fully functional, fully designed spatial data management and application GIS system. Users communicate with the server through the wireless sites to achieve spatial data interaction to enjoy space services. The client of the wireless LBS application platform refers to all kinds of wireless intelligent terminal devices. The application system can 
store some common spatial data on the mobile end to form a mobile database. The common database is SQLite small file database. Mobile databases use a common database interface and wireless integrated application services to seamlessly connect together in a transparent manner, providing users with a variety of wireless services.

As the intermediate layer, the wireless data server layer builds communication bridge for client and server. With the latest technology to expand the coverage of wireless networks, it provides an advanced network access authentication mechanism and encrypts 128 bits of space data to determine the security of the transmission process.

Spatial data is stored by commercial large-scale relational database Oracle, which can easily realize the storage optimization of spatial data and realize the management of database cluster.

This framework plays an important role in practical application, the actual effect is good.

\subsection{Functional Demonstration}

According to the previous design analysis and functional requirements, complete the development of APP. The system successfully invokes the online map to achieve various functions envisioned. The interface is shown in Fig. 3 below.

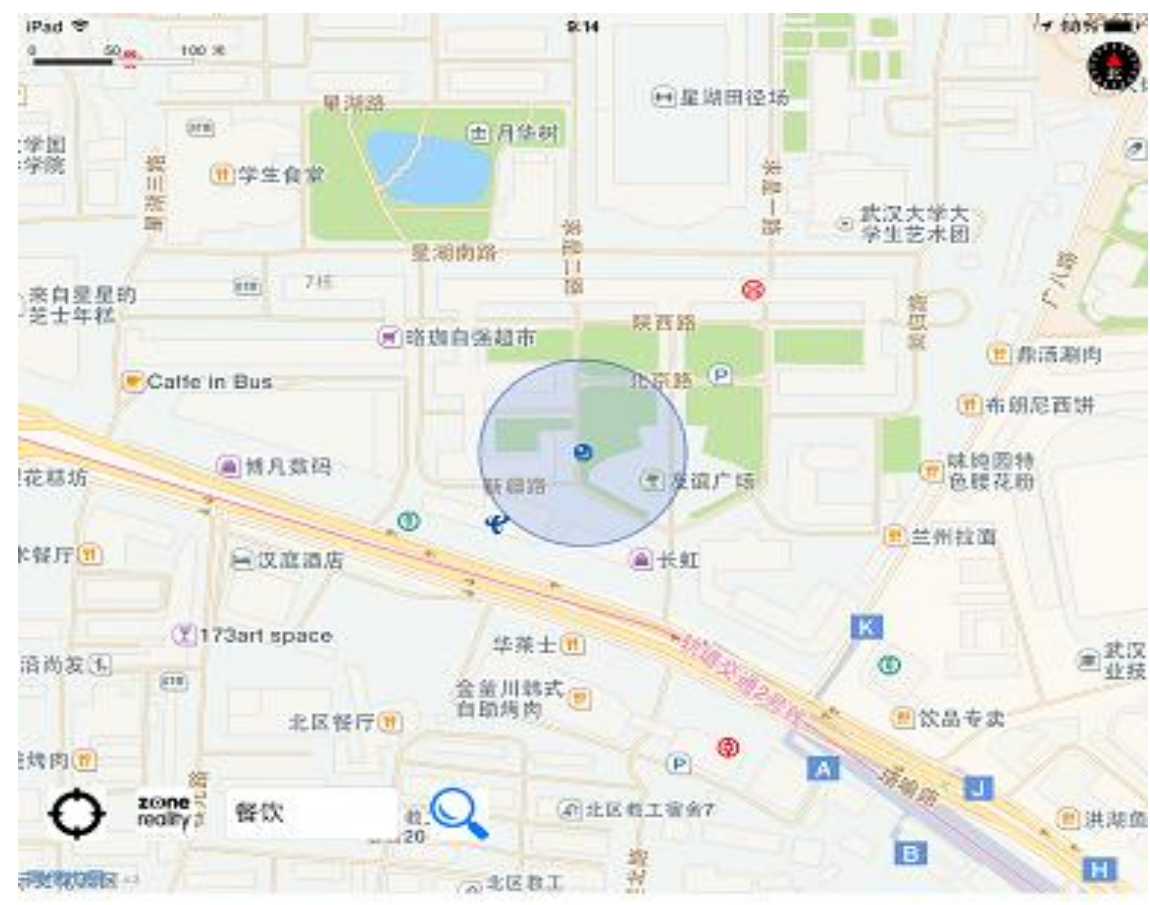

Fig. 3. The interface.

\subsubsection{Positioning and query function}




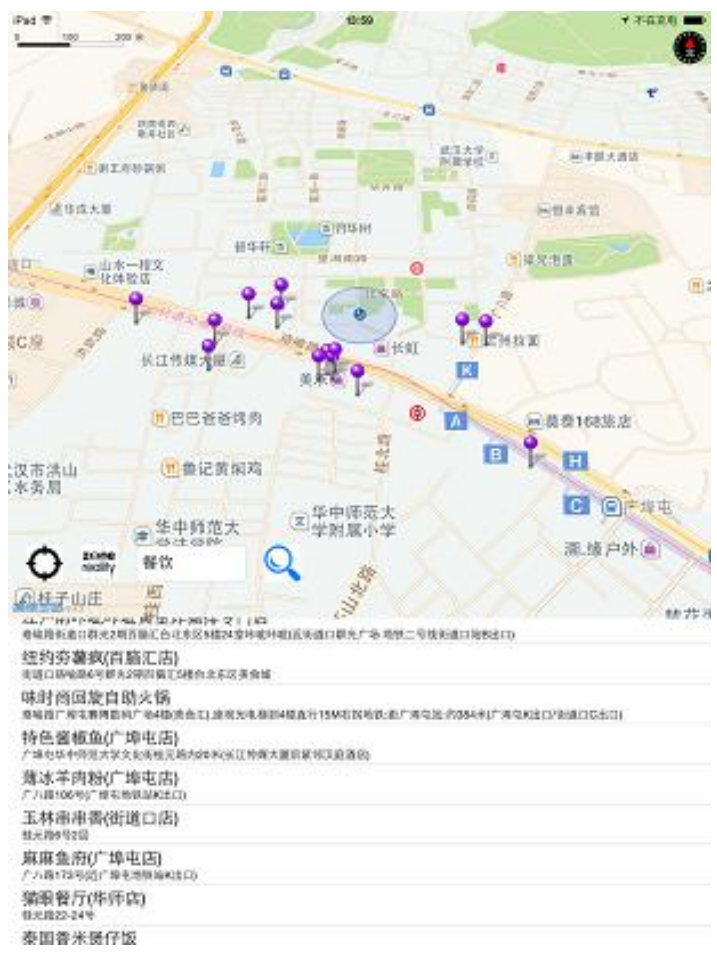

Fig. 4. The POI query.

As shown above, the POI query can be made for the keyword "restaurant" and marked.

\subsubsection{Space target logo display function}

In the lower left corner of the map view, the second button is used for the identification of the target space. After clicking, the system calls the camera to generate camera images. The user changes the direction of the device to capture different targets. As shown in Fig. 5.

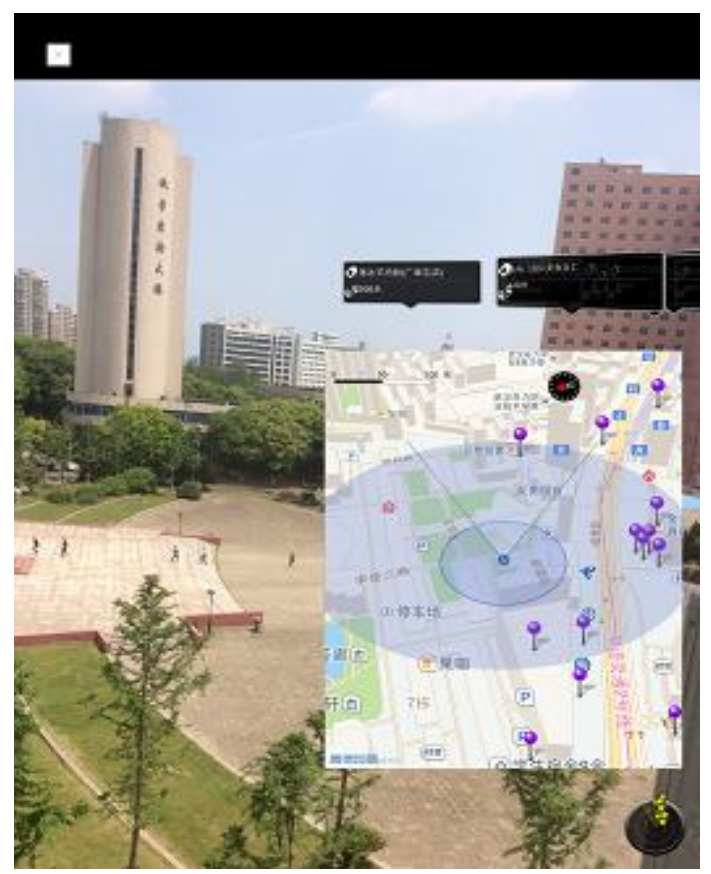

Fig. 5. Live interface.

A space object is displayed on the camera screen, and a space object POI is identified with an air bubble. 
There are three lines of target bubbles. The first row displays the POI name and the distance between the second lines from the location. It can be used to identify different objects at different locations.

The lower right is the radar view of the AR. The yellow stars represent space objects, which are shown in a narrow scale according to the actual spatial distribution. The white fan indicates the camera's area of view. When the device is facing a change, the small star moves. If you enter the white area, you can see it on the map. Click bubbles to see the details.

Path planning can be carried out by long press bubbles. Then, by closing the AR screen, it return to the map and show the map's path on the map.

\section{Conclusion}

The system uses the open source ARKit Augmented Reality(AR) engine, combined with the Amap map 3D API, using Xcode5 as a development tool using OC language, which can realize the real-time identification and dynamic display of the space target location service application system. The use of wireless LBS integrated application platform to achieve spatial data interaction. The system in this paper is on trial, which improves the intuition of space object, which greatly facilitates the acquisition of spatial information. The application of AR technology and real-time display technology to achieve that other similar information systems to provide some technical assistance and recommendations.

\section{Acknowledgment}

The authors thank SHU Shiliang for his assistance and appreciate the reviewers' valuable comments.

\section{References}

[1] Cable, L. P. G. (1999). Method and apparatus for mixing objective-C and C++ objects. U.S. Patent.

[2] Conway, J., \& Hillegass, A. (2012). IOS Programming: The Big Nerd Ranch Guide. Addison-Wesley Professional.

[3] Cox, J., Jones, N., \& Szumski, J. (2012). Professional IOS Network Programming: Connecting the Enterprise to the IPhone and IPad. John Wiley \& Sons, 2012.

[4] Wagner, D., Reitmayr, G., Mulloni, A, et al. (2008). Pose tracking from natural features on mobile phone.

[5] Nilsson, S., Johansson, B., \& Jonsson, A, (2009). Using AR to support cross - Organisational collaboratlon in dynamic tasks..

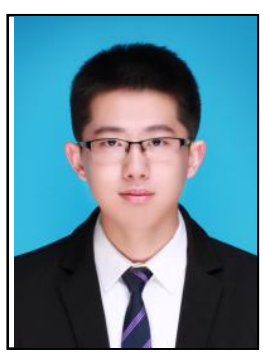

Mingyuan Zhang was born in Shandong, China. He is currently pursuing the bachelor degree in surveying and mapping engineering from Wuhan University. His research interests mainly include data processing of satellite positioning and navigation.

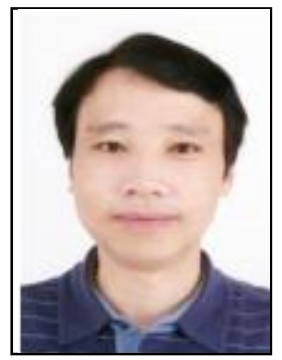

Zhongliang Cai was born in Shandong, China. He received his B.E. and M.S. degree in map making in Wuhan Surveying and Mapping Technology University, China, in 1996 and 1999, respectively. And he received the PhD degree in map making and geographic informatio engineering, School of Resource and Environmental Sciences, Wuhan University, China, 2004. Now he works as a professor in School of Resource and 
Environmental Sciences in WHU and his research interests include digital map theory and method, geographic information service and GIS software developing method.

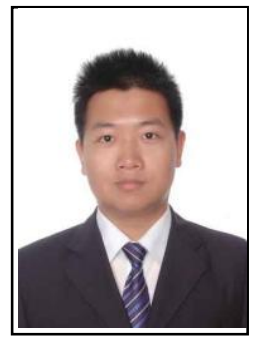

Meng He received his bachelor degree in geographic information system from Wuhan University in 2012 and the M.S. degree in cartographic and geographic information system from Wuhan University in 2015. He now works at Wuhan Kotei Big Data Corporation as a navigation system programmer in Wuhan, China. His research focuses on developing of route planning and route guidance. 